\title{
Comparative Study between Backdoor and Conventional Thyroidectomy in Non-Recurrent Goiter
}

\author{
Yasser Aly El - Sayed, Tamer Yousef Mohamed, Amr Samir Elsaied, Osama Mohammed Mohammed Mostafa* \\ Department of General and Endocrine Surgery, Faculty of Medicine - Mansoura University \\ *Corresponding author: Osama Mohammed Mohammed Mostafa, Mobile: (+20) 01011673775, \\ E-Mail: dr.osama.mans.2020@gmail.com
}

\begin{abstract}
Background: Thyroidectomy is the most common endocrine surgery. The traditional procedure of thyroidectomy is through midline splitting of strap musculatures. The Backdoor approach of thyroidectomy is an established method to explore thyroid and parathyroid lesions. Objective: To evaluate how safe and feasible is the backdoor approach for thyroidectomy and to compare its results with the conventional thyroidectomy technique.

Patients and methods: This prospective study was conducted at Mansoura University Hospitals. We included 40 cases with benign non-recurrent goiter who were divided into 2 equal groups; group "A" included 20 cases who underwent the conventional procedure, and group "B" which included the remaining 20 cases who underwent the backdoor procedure. Results: Regarding the early post-operative period, the backdoor group (group B) experienced less post-operative pain scores and thus, less need for analgesic requirement (1.67 vs. 3.76 for the other group $-\mathrm{p}=$ $0.017)$. One case $(5 \%)$ in group A was complicated by hematoma and was managed via surgical exploration. Both groups had the same range of hospital stay after the operation (2 days). Superficial surgical site infection was diagnosed in one case (5\%) in each group. Transient post-operative hypocalcemia was encountered in 5 cases $(25 \%)$ in group A and 2 cases $(10 \%)$ in group B $(\mathrm{p}=0.179)$. Conclusion: Based on our study findings, it was evident that back door thyroidectomy has more advantages compared to the conventional approach including: Less post-operative pain as peritracheal fascia is not closed, easy identification of parathyroid glands and recurrent laryngeal nerve and avoidance of recurrent laryngeal nerve injuries.
\end{abstract}

Keywords: Backdoor, Conventional Thyroidectomy, Goiter.

\section{INTRODUCTION}

The technique of thyroidectomy has evolved over the last 200 years. The initial contributions made by (Kocher). Over the next years, the procedure has been safe and with less complication (1). Backdoor thyroidectomy uses the same central neck incision however reaches thyroid postero-laterally between anterior borders of sternocleidomastoid (SCM) and strap muscles. It is known as the sternomastoid or "backdoor" or lateral approach (LA) to thyroid (2).

The important structures, which need recognition and preservation during thyroidectomy like parathyroid glands and recurrent laryngeal nerves are posterolaterally situated in relation to thyroid lobe. LA permits an easier access in delivering the gland into operative field for easier recognition of such structures with less retraction and pulling of strap muscles ${ }^{(3)}$. There are only some studies about the effectiveness of LA and the majority of them concluded that LA provided very good visualization of these critical structures ${ }^{(2)}$. Division of cervical strap muscles during thyroidectomy is a controversial issue. Some surgeons do it routinely to gain adequate exposure of the field. Cervical strap muscles have a role in voice pitch control and swallowing functions ${ }^{(4)}$. Some investigators advocate that strap muscles division has no morbid sequelae and recommend its liberal use when operating on large, toxic or neoplastic goiters ${ }^{(5)}$. Strap muscles cutting and reconstitution lead to fibrosis with laryngotracheal fixation that impairs vertical mobility together with strap muscles temporary malfunction with the resulting deleterious effect on voice and swallowing functions ${ }^{(6}$,
7). The respect for the motoricity of cervical strap muscles is necessary not only because they are accessory breathing muscles but more importantly because of their part in voice pitch control and swallowing functions ${ }^{(8,9, \text { and } 10)}$. This study aimed to compare between backdoor and conventional thyroidectomy, as regard feasibility and safety, in nonrecurrent goiters.

\section{PATIENT AND METHODS}

This prospective randomized control study was conducted at Endocrine Surgery Unit, Mansoura University Hospitals. The study group included patients with benign goiters who underwent thyroidectomy between July 2016 and July 2018.

Ethical approval: The study was approved by the local Ethics Committee of Mansoura University.

Written informed consent was taken from all cases in our study after explanation of the aim of the study and explanation of the complications of each approach.

The patients were randomized into 2 groups according to thyroidectomy technique: Group A underwent Conventional Thyroidectomy and Group B underwent backdoor thyroidectomy. Randomization was simple and achieved using the sealed envelope technique.

Inclusion criteria: Simple multinodular goiter, toxic multinodular goiter and solitary thyroid nodule with expected benign criteria.

Exclusion criteria: Huge goiters with retrosternal extensions, recurrent goiters, malignant goiters, history 
of previous neck radiation and unfitness for general anesthesia and surgery.

\section{All patients were submitted to the following:}

1-History taking: Personal history, complaint, present history, past history and family history.

\section{2- Examination:}

General examination: Pulse, blood pressure, temperature, head, eye, chest and extremities examination.

Local examination of the neck:

- Inspection: site, size and shape of swelling.

- Palpation: warmth, tenderness, surface, consistency and mobility

- Percussion: for retrosternal goiter.

- Auscultation: for a bruit.

3- Investigations:

- Laboratory: CBC, blood sugar, INR, SGOT, SGPT, serum creatinine and thyroid function tests (T3, T4, TSH).

- Radiological: Neck U/S, all patients were subjected to US examination with Tirads grading.

- FNAC in solitary thyroid nodule cases.

- Indirect laryngoscopy.

Primary outcome measure:

Identification of external branch of superior laryngeal nerve in both types of thyroidectomy.

\section{Secondary outcome measures:}

Assessment of operative time in minutes from the start of surgery, post-operative pain using the visual analogue scale (VAS) for pain score, blood loss and length of hospital stay. Moreover, conversion rate (from backdoor to conventional), post-operative hypocalcemia (indicated by symptoms, signs and serum Ca level), adhesive scar formation, post-operative surgical site infection and post-operative subcutaneous wound hematoma.

\section{Sample size calculation and power of the study:}

The primary outcome of this study incidence of identification of superior laryngeal nerve. With confidence level of $95 \%$ and type I error of .05, the sample size will be calculated to achieve power of the study of .90 and type II error of .10. The expected incidence of identification of superior laryngeal nerve is $20 \%$ in conventional operation. However, with new approach, the maximum expected incidence of identification is $50 \%$. The calculated sample size was 17 patients in each group. To guard against drop out, the sample size was 20 patients in each group.

\section{Post-operative care:}

All patients were carried to the recovery room postoperatively. After that, they were transferred to the internal ward. Post-operative pain was assessed at (6h,12h, 24h and 48h) using the visual analog scale (VAS) pain score that ranged from Zero (no pain) to ten (worst pain). Unless complications were anticipated, drain was removed and patients were allowed to be discharged usually on the $2^{\text {nd }}$ post-operative day.

Follow-up: That was scheduled at one week, 2 weeks and one month, postoperatively in Endocrine Surgery Unit Outpatient Clinic.
Follow up items: Hypocalcemia (symptoms, signs and serum Ca level), swallowing difficulty, wound infection and voice change.

Post-operative complications: In both procedures were observed during follow up period, clinically and by investigation performed at the scheduled follow up visits (thyroid function, serum Ca total and Ionized, neck US if there is seroma and indirect laryngoscopy if there is voice change).

Signs and symptoms associated with swallowing difficulties: Having pain on swallowing (odynophagia), being unable of swallowing, having sensation of food getting stuck in throat or chest or behind sternum, drooling, hoarse voice, regurgitation and having frequent heartburn and tests (free T3, free T4 and TSH).

\section{Statistical analysis}

Statistical analysis of data was performed by SPSS software version 20 (Chicago, IL). Descriptive data were presented as means with standard deviation or medians with ranges according to data distribution. Categorical variables were presented using frequency distributions. Independent sample t-test was utilized to detect differences in means of continuous variables and Chi-square test was utilized in cases with low expected frequencies. $\mathrm{P}$ values $\leq 0.05$ are considered significant. Of the 246 patients with goiter seen during the study period 40 patients were included in the study.

\section{RESULTS}

All patients completed the follow up period. Finally the analyzed patients 20 in backdoor \& 20 in conventional thyroidectomy. Patients were followed up for 1 - 3 months in conventional and backdoor respectively.

Table (1): Follow up period

\begin{tabular}{|l|c|c|c|}
\hline & $\begin{array}{c}\text { Conventional } \\
\text { thyroidectomy }\end{array}$ & $\begin{array}{c}\text { Backdoor } \\
\text { thyroidectomy }\end{array}$ & $\begin{array}{c}P \\
\text { value }\end{array}$ \\
\hline $\begin{array}{c}\text { Follow up period } \\
\text { (months) } \\
\text { Mean } \pm \text { SD (Range) }\end{array}$ & $\begin{array}{c}1.95 \pm 0.58 \\
(1-3)\end{array}$ & $\begin{array}{c}1.86 \pm 0.62 \\
(1-3)\end{array}$ & 0.638 \\
\hline
\end{tabular}

Patients demographics, the mean age of the included cases was 48.34 and 49.26 years for group $\mathrm{A}$ and $\mathrm{B}$ respectively. Female gender occupied $75 \%$ (15 cases) and $80 \%$ (16 cases) of the study cases for both groups respectively.

Regarding comorbidities, three cases had a history of diabetes mellitus in every group while hypertension was present in 4 cases (20\%) and 3 cases (15\%) in both groups in order of speech. Neither one of these demographics appeared to be significantly different between the study groups ( $p>0.05)$.

Simple multinodular goiter, the most common diagnosis in group A whereas colloid goiter had the same ratio in the other group ( 7 cases $-35 \%$ ). Although study cases showed different degrees of thyroid status, they were medically managed to reach the euthyroid state before operation. Neck US with Tirads grading was done for all cases in this study (Table 2). 
Table (2) Preoperative data.

The age, gender, comorbidities, and preoperative diagnosis were comparable in both group

\begin{tabular}{|c|c|c|c|c|}
\hline & & $\begin{array}{l}\text { Conventional } \\
\text { thyroidectomy }\end{array}$ & $\begin{array}{c}\text { Backdoor } \\
\text { thyroidectomy }\end{array}$ & $P$ value \\
\hline & Age & $48.34 \pm 12.84$ & $49.26 \pm 11.32$ & 0.645 \\
\hline \multirow{2}{*}{ Gender } & -Male & $5(25 \%)$ & $4(20 \%)$ & \multirow{2}{*}{0.739} \\
\hline & -Female & $15(75 \%)$ & $16(80 \%)$ & \\
\hline \multirow{3}{*}{ Comorbidities } & -DM & $3(15 \%)$ & $3(15 \%)$ & \multirow{3}{*}{0.811} \\
\hline & -Hypertension & $4(20 \%)$ & $3(15 \%)$ & \\
\hline & -IHD & $1(5 \%)$ & $1(5 \%)$ & \\
\hline \multirow{5}{*}{ Diagnosis } & -Colloid goiter & $5(25 \%)$ & $5(25 \%)$ & \multirow{5}{*}{0.256} \\
\hline & -Follicular lesion & $3(15 \%)$ & $1(5 \%)$ & \\
\hline & -papillary lesion & $2(10 \%)$ & $5(25 \%)$ & \\
\hline & -SMNG & $7(35 \%)$ & $2(10 \%)$ & \\
\hline & -Toxic goiter & $3(15 \%)$ & & \\
\hline
\end{tabular}

$P$ value were insignificant.

\section{Operative data:}

In group $\mathrm{A}$, the cases were divided equally between two operations; total and hemi-thyroidectomy, that were performed in 10 cases (50\%) for each. On group B, Hemithyroidectomy was performed in 10 cases $(50 \%)$ while 10 cases $(50 \%)$ underwent total thyroidectomy. The backdoor approach had a significantly prolonged operative time when compared to the conventional one (124.72 vs. 102.06 minutes respectively $-\mathrm{p}<0.001)$. No intraoperative complications were encountered in our study. Blood loss was significantly higher for the conventional thyroidectomy group ( 98.06 vs. $80.07 \mathrm{ml}$ for backdoor $-\mathrm{p}=0.026$ ). Although the recurrent laryngeal nerve was identified intraoperatively in all cases, it was easily visualized

and secured in the backdoor approach. Moreover, the superior laryngeal nerve was identified in 15 cases (75\%) in the backdoor group whereas it was identified only 5 cases (25\%) in the conventional group (p 0.009). Additionally, parathyroid glands were identified in all study cases (Table 3).

Table (3) Operative data.

\begin{tabular}{|l|c|c|c|}
\hline & $\begin{array}{c}\text { Conventional } \\
\text { thyroidectomy }\end{array}$ & Backdoor thyroidectomy & P value \\
\hline $\begin{array}{l}\text { Type of operation } \\
\text {-Total thyroidectomy } \\
\text {-Hemithyroidectomy }\end{array}$ & $10(50 \%)$ & $10(50 \%)$ & 1 \\
\hline $\begin{array}{l}\text { Operative time (min) } \\
\text { Mean } \pm \text { SD }\end{array}$ & $102.06 \pm 12.1$ & $124.72 \pm 18.2$ & $<0.001$ \\
\hline Blood loss Nerve & $38.23 \pm 4.19$ & $34.07 \pm 3.55$ & 0.216 \\
\hline $\begin{array}{l}\text { Laryngeal } \\
\text { identification }\end{array}$ & $20(100 \%)$ & & 1 \\
-RLN & $5(25 \%)$ & $20(100 \%)$ & $<0.001$ \\
\hline -SLN & $20(100 \%)$ & $15(75 \%)$ & 1 \\
\hline Parathyroid identification & & $20(100 \%)$ & \\
\hline
\end{tabular}

\section{Post-operative and follow up data:}

Regarding the early post-operative period, the backdoor group (group B) experienced less post-operative pain scores and in turn less analgesic requirement $(1.67 \mathrm{vs.} 3.76$ for the other group $-\mathrm{p}=0.017)$. One case $(5 \%)$ in group A was complicated by hematoma and was managed via surgical exploration. Both groups had the same range of hospital stay after the operation (2 days). Superficial surgical site infection was diagnosed in one case (5\%) in each group. Transient post-operative hypocalcemia was encountered in 5 cases $(25 \%)$ in group A and 2 cases $(10 \%)$ in group B ( $\mathrm{p}=0.179)$. Swallowing difficulty was experienced by 6 cases $(30 \%)$ in group A and only one case (5\%) in the other group $(\mathrm{p}=0.027)$. This can be explained by the presence of adherent scar that was detected more in the conventional thyroidectomy group. Post-operative neck paresthesia was not detected during the post-operative period, neither during the follow up. Post-operative seroma was detected in five cases $(25 \%)$ in group A whereas only 2 cases $(10 \%)$ in the other group had the same complication $(\mathrm{p}=0.179)$. Additionally, transient hoarseness of voice was experienced by 6 patients (30\%) in group A, while only experienced in 2 cases $(10 \%)$ in group $\mathrm{B}(\mathrm{p}=0.037)$ as shown in table (4). 
Table (4) Post-operative and follow up data.

\begin{tabular}{|l|c|c|c|}
\hline & $\begin{array}{c}\text { Conventional } \\
\text { thyroidectomy }\end{array}$ & $\begin{array}{c}\text { Backdoor } \\
\text { thyroidectomy }\end{array}$ & P value \\
\hline $\begin{array}{l}\text { Follow up period/months } \\
\text { Mean } \pm \text { SD }\end{array}$ & $\begin{array}{c}1.95 \pm 0.58 \\
(1-3)\end{array}$ & $\begin{array}{c}1.86 \pm 0.62 \\
(1-3)\end{array}$ & 0.638 \\
\hline Pain score after 6 h & $3.76 \pm 0.72$ & $1.67 \pm 0.62$ & 0.017 \\
\hline Pain score after 12 h & $2.39 \pm 0.50$ & $1.58 \pm 0.32$ & $0.003^{*}$ \\
\hline Pain score after 24 h & $2.26 \pm 0.44$ & $1.45 \pm 0.28$ & $0.001^{*}$ \\
\hline Pain score after 48 h & $2.18 \pm 0.12$ & $1.12 \pm 0.22$ & $0.001^{*}$ \\
\hline \multicolumn{3}{|c|}{ Hypocalcemia } \\
\hline Temporary & $5(25 \%)$ & $2(10 \%)$ & 0.179 \\
\hline Permenant & $1(5 \%)$ & $0(0 \%)$ & 0.847 \\
\hline Swallowing difficulty & $6(30 \%)$ & $1(5 \%)$ & 0.027 \\
\hline Hematoma & $1(5 \%)$ & $0(0 \%)$ & 0.847 \\
\hline Seroma & $2(10 \%)$ & $1(5 \%)$ & 0.456 \\
\hline Wound infection & $0(0 \%)$ & $0(0 \%)$ & 0.456 \\
\hline $\begin{array}{l}\text { Voice change due to RLN } \\
\text { affection }\end{array}$ & $2(10 \%)$ & $1(5 \%)$ & 1 \\
\hline
\end{tabular}

\section{DISCUSSION}

In our study, the mean age of the included cases was 48.34 and 49.26 years for conventional and backdoor groups respectively. No significant difference was detected between the two groups regarding that parameter $(p=0.645)$. In the current study, females represented 75 and $80 \%$ of cases in both groups respectively. Gender was not statistically different between the two groups $(\mathrm{p}=0.739)$. Another study reported that females represented 92 and $80 \%$ of cases in the conventional and backdoor groups respectively. Like our study, there was no significant difference between the two groups regarding gender ${ }^{(3)}$.

Regarding the indication of surgery in the current study, SMNG was the commonest indication in the conventional group (35\%), whereas colloid goiter was the commonest in the backdoor group (35\%). Other indications included follicular, papillary, and toxic goiter. Ramouz et al. ${ }^{(11)}$ reported that MNG was the commonest indication of thyroidectomy in their study (43.4\% and $52.5 \%$ respectively), followed by papillary lesions (26.6 and $24.9 \%$ respectively). Other causes included follicular lesions, Grave disease, thyroid adenoma, Hashimoto thyroiditis and thyroid cyst.

As regards the type of thyroidectomy performed, it was not significantly different between the two study groups $(\mathrm{p}=0.229)$. Total thyroidectomy was performed in 50 and $35 \%$ of cases in both groups respectively, while the remaining cases underwent hemithyroidectomy. Singaporewalla et al. (2) study reported that hemithyroidectomy was performed in $40 \%$ of cases in both groups. Moreover, total thyroidectomy was done in 40 and $44.4 \%$ of cases in both groups respectively. The remaining cases underwent total thyroidectomy with lymph node dissection. The type of operation showed no significant variable between the two groups $(p=0.837)$. This is consistent with our study results. However, lymph node dissection was not performed in our study as malignant cases were excluded.

Coming to the operative time in the current study, it was significantly prolonged in the backdoor group (124.72 vs. 102.06 minutes $-\mathrm{p}<0.001$ ). ElErian et al. ${ }^{(12)}$ study reported that the mean operative time of backdoor procedure was 90 minutes (range, 60 - 140). On the contrary, another study reported no significant difference between the two groups regarding operative time $(\mathrm{p}=0.984)$ although it was longer in the conventional group. It had a mean of 137 and 124 minutes in both groups respectively ${ }^{(2)}$.

Regarding blood loss in the current study, it was significantly lower in the backdoor group $(\mathrm{p}=$ 0.026 ). It had a mean of 98.23 and $80.07 \mathrm{ml}$ in the conventional and backdoor groups respectively. Elkordy et $\boldsymbol{~ a l . ~}{ }^{(3)}$ study reported that the mean amount of intraoperative blood loss was significantly lower in the backdoor group (70 vs. $120 \mathrm{ml}$ for the conventional procedure $-\mathrm{p}=0.009$ ). This comes in line with our results. Other author reported that the mean amount of blood loss was $70 \mathrm{ml}$ (range, $45-140 \mathrm{ml}{ }^{(\mathbf{1 2})}$. This also agrees with our findings regarding the backdoor approach.

In the current study, although identification of recurrent laryngeal nerves and parathyroids did not differ statistically between the two groups ( $p>0.05)$, superior laryngeal nerve was more frequently identified in the backdoor group (65\% vs. $35 \%$ of cases $-p=$ 0.013). Most surgeons believe that backdoor approach enables easier access to posterolateral surface of thyroid where important structures like parathyroid and nerves exist. In addition, backdoor approach enables easier delivery of large goitre with no necessity for transection 
of strap muscles horizontally (13, 14). Another study reported that recurrent laryngeal nerve, superior laryngeal nerve, and parathyroids were identified in 93 , 30 , and $100 \%$ of cases respectively using the backdoor approach ${ }^{(\mathbf{1 2})}$.

In the current study, analysis of postoperative pain according to VAS score revealed significant lower scores in the backdoor group compared to the conventional procedure (1.67 vs. $3.67-\mathrm{p}=0.017$ ).

Similarly, another study confirms our findings regarding postoperative pain. It was significant lower in the backdoor group $(\mathrm{p}=0.02)$. VAS score had a mean of 1.86 and 0.82 in both groups respectively ${ }^{(2)}$. The excessive retraction of strap muscles laterally in the conventional approach, in order to perform dissection postero-laterally along the thyroid lobe, could also be the possible reason for increased pain score in these patients when delivering large glands ${ }^{(2)}$. Conversely, another study reported that pain was well tolerated between the two groups after surgery ${ }^{(3)}$. However, that study did not use VAS score to assess post-operative pain like ours.

Regarding swallowing problems, it was significantly less encountered in the backdoor group (5 vs. $30 \%$ of cases in the conventional group $-\mathrm{p}=0.027$ ). Similarly, voice changes were significantly less experienced in the backdoor cases (10 vs. $30 \%$ of cases in the conventional group $-\mathrm{p}=0.037$ ). Kim in his study mentioned that handling of strap muscles and reconstitution or even excessive retraction may affect voice quality and swallowing function postthyroidectomy ${ }^{(4)}$.

In the current study, the incidence of hypocalcemia did not differ between the two groups ( $\mathrm{p}$ $=0.179$ ). It occurred in $25 \%$ and $10 \%$ of cases in both groups respectively. El-kordy et al. ${ }^{(3)}$ study confirmed our findings regarding the incidence of hypocalcemia after operation. It occurred in 22.7 and $34.1 \%$ of cases in the conventional and backdoor groups respectively. Like our results, no significant difference between both groups was detected $(\mathrm{p}=0.345)$.

The hospital stay did not differ between the two groups $(\mathrm{p}=1)$ in the study groups. It had a median of 2 days in both groups. Another Egyptian study reported that the mean period of hospital stay was 3 days for the backdoor approach (range, $2-6)^{(12)}$. On the contrary, El-kordy et al. ${ }^{(3)}$ reported that the hospital stay was significantly longer for backdoor cases (3 vs. 2 days for the conventional procedure $-\mathrm{p}=0.016$ ). This disagrees with our findings.

In the current study, the incidence of wound hematoma or seroma did not differ between the two study groups ( $\mathrm{p}=0.847$ and 0.179 respectively). Another study reported that hematoma/seroma formation was detected in $7 \%$ of backdoor thyroidectomy cases ${ }^{(\mathbf{1 2})}$.
Another important advantage of backdoor approach is that a gap was left between anterior border of sternomastoid and lateral end of strap muscles after thyroidectomy. Such gap is always left open to act as a safety valve in the event patient had a massive postoperative hemorrhage. The blood could easily flow through this gap superficially below skin flap to prevent severe tracheal compression, laryngeal oedema and stridor. In conventional approach, midline reapproximation of strap muscles and the lack of space between sternomastoid and lateral edge of strap muscles might cause a life-threatening haematoma under strap muscles ${ }^{(2)}$.

Regarding the incidence of surgical site infections, it did not differ between the two groups (5\% of cases in each group $-p=1$ ). Unfortunately, previous reports about backdoor procedure did not report the incidence of surgical site infections with the procedure $(2,3)$.

\section{CONCLUSION}

Based on our study findings, it was evident that back door thyroidectomy has more advantages compared to the conventional approach including:

- Avoidance of adherent scar formation in the peritracheal fascia.

- Easy exposure of the upper thyroid pole.

- Easy ligation of superior thyroid artery.

- Avoidance of tension hematoma formation as the cave is not closed.

- Less post-operative pain as peritracheal fascia is not closed.

- Easy identification of parathyroid glands and recurrent laryngeal nerve.

- Avoidance of recurrent laryngeal nerve injuries.

- Smaller skin incision.

\section{REFERENCES}

1. Shaha A (2019): Thyroidectomy in the Eldery. Eur J Surg Oncol., 45 (3): 299-300.

2. Singaporewalla R, Tan B, Rao A (2018): The lateral "backdoor" approach to open thyroid surgery: A comparative study. Asian Journal of Surgery, 41 (4): 384388.

3. El-kordy M, Attia S, Anwar H et al. (2019): Lateral backdoor approach versus conventional anterior approach in recurrent Thyroid surgery. Journal of Advanced Pharmacy Education \& Research, 9 (1): 6-10.

4. Kim Y, Cho J, Kim C et al. (2000): Voice Comparison between Strap Muscle Retraction and Cutting Technique in Thyroidectomy. Korean Journal of OtorhinolaryngologyHead and Neck Surgery, 43 (9): 985-991.

5. Henry L, Solomon N, Howard $R$ et al. (2008): The functional impact on voice of sternothyroid muscle division during thyroidectomy. Ann Surg Oncol., 15 (7): 2027-2033.

6. Sinagra, D, Montesinos, M, Tacchi V et al. (2004): Voice changes after thyroidectomy without recurrent laryngeal 
nerve injury. Journal of the American College of Surgeons, 199 (4): 556-560.

7. Lombardi C, Raffaelli M, D'Alatri L et al. (2006): Voice and swallowing changes after thyroidectomy in patients without inferior laryngeal nerve injuries. Surgery, 140 (6): 1026-1034.

8. Stojadinovic A, Shaha A, Orlikoff $\mathrm{R}$ et al. (2002): Prospective functional voice assessment in patients undergoing thyroid surgery. Ann Surg., 236 (6): 823-832.

9. Jaffe V, Young A (1993): Strap muscles in thyroid surgery: to cut or not to cut?. Annals of the Royal College of Surgeons of England, 75 (2): 118-125.

10. McIvor N, Flint D, Gillibrand J et al. (2000): Thyroid surgery and voice-related outcomes. Aust N Z J Surg., 70 (3): 179-183.

11. Ramouz A, Rasihashemi S, Safaeiyan A et al. (2018): Comparing postoperative complication of LigaSure Small
Jaw instrument with clamp and tie method in thyroidectomy patients: a randomized controlled trial [IRCT2014010516077N1]. World J Surg Oncol., 16 (1): 154-158.

12.El-Erian A, El-raouf A, Nabeel I et al. (2015): Lateral approach to attack superior thyroid vascular pedicle eliminates the need for strap muscles cutting during thyroidectomy. Endocrine and Oncologic Surgery, 83: 125-134.

13. Sebag F, Palazzo F, Harding J et al. (2006): Endoscopic lateral approach thyroid lobectomy: safe evolution from endoscopic parathyroidectomy. World J Surg., 30 (5): 802805.

14. Kiriakopoulos A, Dimitrios T, Dimitrios L (2004): Use of a diathermy system in thyroid surgery. Arch Surg., 139 (9): 997-1000. 